\title{
Update on Peptic Ulcers in the Pediatric Age
}

\author{
Graziella Guariso and Marco Gasparetto \\ Unit of Gastroenterology, Digestive Endoscopy, Hepatology, and Care of the Child with Liver Transplantation, \\ Department of Woman and Child Health, University Hospital of Padova, Via Giustiniani 2, 35128 Padova, Italy
}

Correspondence should be addressed to Marco Gasparetto, markgasp@gmail.com

Received 11 August 2012; Accepted 4 September 2012

Academic Editor: Joachim Labenz

Copyright ( 2012 G. Guariso and M. Gasparetto. This is an open access article distributed under the Creative Commons Attribution License, which permits unrestricted use, distribution, and reproduction in any medium, provided the original work is properly cited.

\begin{abstract}
Background. Peptic ulcer disease (PUD) in children is reported worldwide, although it is relatively rare as compared with adults. Helicobacter pylori (HP) infection is a common cause of PUD in the pediatric age. Other risk factors include the use of nonsteroidal anti-inflammatory agents (NSAIDs), steroids, immunosuppressive drugs, and stressful events. Aim. To critically review the evidence on epidemiology, diagnostic management, and available treatments for PUD in the pediatric age. Methods. A MEDLINE search was performed indicating keywords as "Peptic Ulcer Disease," "Epidemiology," "Pediatric," "Helicobacter pylori," "Gastric ulcer," "Bulbar Ulcer," and "Upper Gastrointestinal Bleeding." A selection of clinical trials, systematic reviews, and meta-analyses within the time period 2002-2012 was performed. Results. PUD in children is reported worldwide with an estimated frequency of $8.1 \%$ in Europe and of $17.4 \%$ in the US. When the underlying cause of PUD is addressed, the prognosis is excellent. Standard triple therapy, bismuth-based quadruple therapy, and the sequential therapy represent the current recommended treatments for HP related ulcers. NSAIDs related ulcers are treated by stopping the causative medications and by administration of proton-pump inhibitors or antisecretory drugs. Conclusions. PUD still represents a major concern in the paediatric age. A careful differential diagnosis and an adequate treatment constitute an excellent prognosis.
\end{abstract}

\section{Introduction}

Peptic ulcers are discontinuities of the gastric or duodenal mucosa with penetration to the muscularis mucosae and exposure of the submucosa $[1,2]$.

Primitive ulcers are caused by alterations of the gastric function (i.e., increased $\mathrm{HCl}$ production and pepsin function); they are mainly single lesions and are usually found at the small gastric curve and at the antrum.

Secondary ulcers, on the contrary, are caused by extragastric pathogenic events, that is, stress or drugs. They can be multiple and can have a spread localization within the stomach.

More than $20 \%$ of patients have a family history of duodenal ulcers. In up to one third of patients with duodenal ulcers, basal acid output (BAO) and maximal acid output are increased. A study by Schubert and Peura [3] attested that individuals are at especially high risk those with a basal acid production (BAP) greater than $15 \mathrm{mEq} / \mathrm{h}$. In addition to the increased gastric and duodenal acidity observed in some patients with duodenal ulcers, accelerated gastric emptying is also often present.

A common cause of peptic ulcers in the pediatric age is Helicobacter pylori (HP) infection [4-6], though the use of nonsteroidal anti-inflammatory agents (NSAIDs), like aspirin and ibuprofen, represents a significant cause of ulcers as well [7].

Other treatments comprehending steroids and antineoplastic and immunosuppressive drugs can be causative of peptic ulcers. A reduction of the protective effect of prostaglandins on gastric mucosa is considered to be the main pathogenic mechanism.

Other stressful events (i.e., shock, sepsis, burnings, major trauma, intracranial hypertension, surgical procedures, and chronic diseases) can provoke acute gastric ulcers, also in the pediatric age [8]. Lesions generally appear 3-6 days after the event and the main related symptoms are bleeding and 
abdominal pain. Most of times, multiple lesions involving any part of the stomach are observed.

Cushing ulcers are associated with a brain tumor or injury; they are typically single, deep ulcers located in the duodenum or stomach that are prone to perforation.

Stress and spicy foods do not cause ulcers, but can exacerbate existing ones. Obesity has been shown to have an association with PUD, and patients should be counseled regarding benefits of weight loss.

Children presenting serious medical conditions may develop peptic ulcers as a secondary condition. Neoplastic ulcers are instead related to development of lymphomas.

Hypersecretory states that may uncommonly cause peptic ulcer disease (PUD) include gastrinoma (ZollingerEllison syndrome), multiple endocrine neoplasia type I (MEN-I), antral G-cell hyperplasia, systemic mastocytosis, basophilic leukemias, cystic fibrosis, short bowel syndrome, and hyperparathyroidism.

The most significant ulcer related symptoms involve dull stomach ache or pain, intermittent abdominal discomfort (typically occurring several hours after a meal or in an empty stomach, often being relieved by eating), gas and bloating, nausea and, less commonly, vomiting [9].

Gastric and duodenal ulcers cannot be differentiated based on history alone, although some findings may be suggestive. Epigastric pain is the most common symptom of both gastric and duodenal ulcers and is characterized by a gnawing or burning sensation occurring shortly after meals with gastric ulcer and 2-3 hours afterward with duodenal ulcer [9].

Alarm symptoms supporting the suspect of GI bleeding or ulcer perforation involve sudden, sharp abdominal pain, black or bloody stools, and bloody or coffee-like vomits.

Other alarm features which warrant prompt gastroenterology referral include anemia, early satiety, unexplained weight loss, progressive dysphagia or odynophagia, and family history of GI cancer [9].

Diagnostic examinations include urease test, stool tests for HP detection, and upper-gastrointestinal (GI) tract endoscopy [9].

In most patients with uncomplicated PUD, routine laboratory tests are usually unhelpful; only a combined evaluation of serum pepsinogens A (PGA) and C (PGC), gastrin-17 (G17), and anti-HP antibodies (GastroPanel) [10] has been proposed as a useful blood test to investigate HP-associated gastric mucosal inflammation.

Plasma contains two main gastrin isoforms: gastrin 34 (G34, large gastrin) and gastrin 17 (G17, small gastrin). G17 accounts for about 95\% of antral gastrin, while G34 accounts for about $60 \%$ of duodenal gastrin. When the gastric mucosa is inflamed, its secretory function may be significantly altered. HP may enhance gastrin release through bacterial urease catalysing the production of ammonia which acts directly on gastrin cells, as well as through the proinflammatory cytokines IL-8, IL-2, and TNF- $\alpha$ which enhance gastrin secretion [10].

Since endoscopy is not recommended as a first diagnostic approach for children with abdominal pain without alarm symptoms and since it cannot be performed in every centre,
GastroPanel appears to be useful as a non invasive biochemical test, to support the decision to perform or not uppergastrointestinal endoscopy, thus to optimize the healthcare resources. The serum determination of PGA, PGC, and of their derived index, PGA/PGC, associated with G17 and anti-HP antibodies allows a more accurate characterization of the aetiology (whether or not associated with HP) and topography (antral and/or corpus predominant) of gastritis [10].

Negative test results should allow pediatricians to reasonably rule out the presence of major gastroduodenal diseases and therefore to avoid endoscopy [10].

Rapid urease tests are considered the test of choice to detect HP; among noninvasive tests, fecal antigen testing is more accurate than antibody testing [11].

Upper-GI endoscopy is the preferred diagnostic test for the evaluation of patients with suspected PUD, consenting to detect the presence and degree of active bleeding, and to attempt hemostasis by direct measures, if required. A secondary role is currently represented by the upper-GI-tract $\mathrm{X}$-ray [9].

The 2011 Evidence-based Guidelines from ESPGHAN and NASPGHAN for HP infection in children are based on a systematic revision of the literature referring to the time period 2000-2007 [12]. An evidence grade was assigned according to the classification system of the Oxford Centre for Evidence Based Medicine, and 490 out of 2042 papers were included.

Within the pediatric population, HP related gastrointestinal symptoms are generally aspecific; as a consequence the primary goal of clinical investigation of gastrointestinal symptoms is to determine the underlying cause of the symptoms and not solely the presence of $H$. pylori infection. Diagnostic testing for $H$. pylori infection is not recommended in children with functional abdominal pain, whereas it can be indicated in symptomatic children with first grade relatives affected by gastric cancer, as well as in those children with refractory iron-deficient anaemia once the other possible differential diagnoses have been excluded. There is no sufficient evidence attesting any correlation between HP and otitis media, upper respiratory ways infections, periodontal diseases, food allergies, idiopathic thrombocytopenic purpura, and short stature [12].

Sensibility is of $75 \%-100 \%$ for urease rapid tests and of $66 \%-100 \%$ for histopatologic HP detection.

13C-urea breath test (UBT) has high sensibility and specificity, thus representing a reliable noninvasive test to detect the success of HP eradication. A false negative can be determined by increases in gastric $\mathrm{pH}$ which diminish urease activity. False positives can be observed when the marker is degraded by intestinal flora (especially in children aging $<6$ years) or when low distribution volumes determine a diminished $\mathrm{CO}_{2}$ production rate. Sources of urease rapid test variability include age, marker type and dosage, meal type, pretest fasting, detection timing, type of analysis, and cut-off levels [12].

HP faecal antigen detection is a non invasive test which can be reliable to determine the success of HP eradication. Detection techniques on faecal antigen include monoclonal 
and polyclonal EIA as well as immune-chromatographic tests. Advantages of this technique are that it is not agedependant, it is more easy to be performed on children, and less expensive. Moreover, anti-HP antibodies are resistant to degradation [12].

The detection of anti-HP antibodies (IgG, IgA) on blood, serum, urines, and saliva is not demonstrated to be a reliable test for clinical purposes [12].

HP culture has a low sensibility, whereas specificity is of $100 \%$.

As a summary about HP gastritis diagnosis according to the current available evidences, it is recommended that the initial diagnosis of HP infection be based on either positive histopathology and positive rapid urease test or a positive culture. The following combined findings are required [12]:

(i) histopathology + urease rapid test,

(ii) invasive test + noninvasive test,

(iii) HP culture alone.

In order to correctly perform invasive and non invasive tests for HP detection, the following treatment suspensions are required [12]:

(i) proton pump inhibitors (PPIs) for at least 2 weeks,

(ii) antibiotics for at least 4 weeks.

The Forrest classification describes four types of peptic ulcers, based on the endoscopic characteristics of the associated upper gastrointestinal hemorrhage [13] as follows:

(i) Forrest Ia: spurting arterial bleeding;

(ii) Forrest Ib: oozing arterial hemorrhage;

(iii) Forrest IIa: large nonbleeding visible vessels;

(iv) Forrest IIb: adherent clot;

(v) Forrest IIc: hematin on ulcer base;

(vi) Forrest III: lesions without signs of recent hemorrhage.

Benign ulcers are endoscopically characterized by mild dimensions, oval or roundish shape, and a whitish-grey base, consisting in covering fibrin and granulation tissue. Borders are thin and plane. A basal vase can be detected and represent a source of hemorrhage. Gastric plicae are usually converging to the ulcer.

Malignant ulcers generally present an irregular base, with necrotic base covered by fibrin. However, histological examination only can differentiate the two ulcer types.

\section{Aim and Methods}

This paper is conceived to critically review evidence on epidemiology, diagnostic management, and available treatments for PUD in the pediatric age. A MEDLINE search was performed indicating key-words as "Peptic Ulcer Disease," "Epidemiology," "Pediatric," "Helicobacter pylori," "Gastric ulcer," and "Bulbar Ulcer." A selection of relevant English-language clinical trials, systematic reviews, and meta-analyses within the last ten years (time period 2002-2012) were performed. Previous references and case reports were also included when basically supporting the first selection. PubMed, Cochrane Library, Trip Database, Ovid Medicine, Medscape Reference, and Up To Date were used as referral databases. Jadad Scale (score 0 to 5) was used as a predetermined strategy of inclusion for clinical trials.

\subsection{Epidemiology of Peptic Ulcer Disease Worldwide in the} Pediatric Population. PUD in children is reported worldwide (Table 1), although being relatively rare as compared with adults [14].

Solid data attesting the frequency of PUD during childhood in Europe have recently been described by a European multicenter study [15] by which the role of known risk factors within the studied population was also analyzed. Although limited by the short-time duration and the heterogeneity of the 694 children included throughout 19 European centres, this study shows a frequency of $8.1 \%$ of ulcers and/or erosions in children, occurring mainly in the second decade of life. HP infection and gastrotoxic medications were less frequently implicated than expected. More than one risk factor was detected in $57 \%$ of the children with PUD: HP infection was present in $27 \%$ of these children, where NSAIDs were used in 14\%, H2-blockers in $10 \%$, antibiotics in $10 \%$, steroids in $9 \%$, immunesuppressive drugs in $9 \%$, and proton-pump inhibitors in $9 \%$ of the children. No risk factors were observed in $43 \%$ of the enrolled children. The main indications for endoscopy were epigastric or abdominal pain and suspicion of gastroesophageal reflux disease. Epigastric tenderness, hematemesis, melena, and weight stagnation were significantly associated with ulcers/erosions, whereas sex, HP infection, and socioeconomic and lifestyle factors were equally distributed.

As regards the US, an epidemiologic study by Brown et al. [16] aimed to determine the incidence of peptic ulcer bleeding in pediatric patients, analyzing a hospital inpatient database and an insurance claims database. Using data from the inpatient database, the total number of cases of hospitalization of pediatric patients for PUD bleeding in the US in 2008 was estimated to be between 378 and 652 . This translated to an incidence of 0.5 to $0.9 / 100.000$ individuals in the pediatric population. Using data from the insurance claims database, the incidence of PUB was estimated to be 4.4/100.000 individuals. Overall, $17.4 \%$ of insured pediatric patients diagnosed as having any upper gastrointestinal ulcer in 2008 were reported to have developed PUB. In conclusion, the estimated incidence of PUB in the US pediatric population in 2008 ranged from 0.5 to $4.4 / 100.000$ individuals.

HP infection currently represents the main cause of peptic ulcer in children, influencing diagnostic and treatment procedures $[8-16,19,20]$. HP is mainly acquired in childhood and is particularly frequent in developing countries [21].

The evidence for a vertical HP transmission is described in the review by Sýkora and Rowland, which analyzes the main studies about HP infection between April 2010 and 
TABLE 1

\begin{tabular}{|c|c|c|c|c|c|c|}
\hline Author/yr/country & Study methods & $\begin{array}{l}\text { Number of } \\
\text { children } \\
\text { examined }\end{array}$ & PUD & PUB & HP infection & NSAID use \\
\hline $\begin{array}{l}\text { Kalach et al. [15] } \\
\text { 2010; Europe }\end{array}$ & $\begin{array}{l}\text { Multicenter, } \\
\text { prospective }\end{array}$ & 694 & $56 / 694(8 \%)$ & n.d.a. & $15 / 56(27 \%)$ & $8 / 56(14 \%)$ \\
\hline $\begin{array}{l}\text { Brown et al. [16] } \\
\text { 2012; US }\end{array}$ & $\begin{array}{l}\text { Multicenter } \\
\text { (on national } \\
\text { registries), } \\
\text { retrospective }\end{array}$ & 29.3 million & 7280 & $\begin{array}{c}1267(17.4 \% \text { of } \\
\text { PUD) }\end{array}$ & n.d.a. & n.d.a. \\
\hline $\begin{array}{l}\text { Shu-Ching et al. } \\
\text { [14] 2009; Taiwan }\end{array}$ & $\begin{array}{l}\text { Monocenter, } \\
\text { retrospective, and } \\
\text { prospective }\end{array}$ & 1234 & $\begin{array}{l}67(5.4 \%) \\
\text { gastric } 27(40.3 \%) \\
\text { duodenal } 40(59.7 \%)\end{array}$ & $43(64.2 \%$ of PUD $)$ & $32(47.7 \%)$ & $11(16.5 \%)$ \\
\hline $\begin{array}{l}\text { Egbaria et al. [17] } \\
\text { 2008; Israel }\end{array}$ & $\begin{array}{l}\text { Monocenter, } \\
\text { retrospective }\end{array}$ & 751 & $\begin{array}{l}169(22.5 \%) \\
\text { (i) ulcers } 51(6.8 \%) \text {, } \\
\text { erosions } 118(15.7 \%) \text {; } \\
\text { (ii) gastric } 124(73.3 \%) \text {, } \\
\text { duodenal } 58(34.3 \%)\end{array}$ & $4(2.4 \%$ of PUD $)$ & $112(66.3 \%)$ & $34(20.1 \%)$ \\
\hline $\begin{array}{l}\text { El Mouzan and } \\
\text { abdullah [18] } \\
\text { 2004; Saudi Arabia }\end{array}$ & $\begin{array}{l}\text { Monocenter, } \\
\text { retrospective, and } \\
\text { prospective }\end{array}$ & 521 & $\begin{array}{l}24(5 \%) \\
\text { gastric } 4(17 \%), \\
\text { duodenal } 20(92 \%)\end{array}$ & 5 (21\% of PUD) & $13 / 15(87 \%)$ & nda \\
\hline
\end{tabular}

yr: year; PUD: peptic ulcer disease; PUB: peptic ulcer bleeding; HP: Helicobacter pylori; NSAID: nonsteroidal anti-inflammatory drugs; n.d.a.: no data available.

March 2011 [22]. HP infection was associated with chronic gastritis in all infected children, but only a subgroup of them developed PUD [22]. The discovery of a potential role for jhp0562, the gene which encodes for the cell envelope protein glycosyltransferase, in the progression to PUD is also a key step in distinguishing children at risk of PUD from those who are not, and so determining those who require treatment to eradicate HP.

As there are no well-established criteria leading directly to diagnosis, the authors conclude that pediatricians should include HP infection and PUD in the differential diagnosis list when evaluating children with abdominal pain, failure to thrive, and upper gastrointestinal system bleeding [22].

A Turkish study by Uğraş and Pehlivanoğlu detected PUD in $13.2 \%$ of children who underwent endoscopic examination. In the studied population, peptic ulcers were mostly gastric and HP positive, and most of the children were admitted due to abdominal pain [21].

Unfortunately, high levels of HP treatment failure continue to be reported, and there remains an urgent need for more effective HP eradication regimes for children [22].

Moreover, the rise in non-HP-associated ulcers and erosions continues to be reported with no apparent risk factors for these ulcers identified to date [22].

Although the prevalence of NSAID gastropathy in children is unknown, it seems to be increasing, especially in children with chronic arthritis treated with NSAIDs. Case reports have demonstrated gastric ulceration from low-dose ibuprofen in children, even after just 1 or 2 doses [23].

A recent study from Taiwan by Shu-Ching et al. [14] aimed to assess the etiology, clinical, and histological characteristics and treatment of PUD in children. From 1234 endoscopic examinations, $5.4 \%$ of children presented gastric ulcer (GU) or duodenal ulcer (DU): $47.7 \%$ of these children had HP infection and $16.5 \%$ had previous use of NSAIDs. Non-HP, non-NSAID PUD was found in $35.8 \%$ of patients. Children with HP-related PUD had a significantly higher mean age, antral chronic inflammatory score, rate of familial PUD, and presence of DU and nodular gastritis than those with NSAID-related PUD and with non-HP, non-NSAID PUD. In contrast, children with NSAID-related PUD had a higher rate of upper gastrointestinal bleeding, associated with acute febrile disease, than those with HP-related and non-HP, non-NSAID PUD. All but two patients with nonHP, non-NSAID PUD were disease-free after HP eradication and proton pump inhibitor treatment for 1-2 months. These data from Taiwan concluded that in children, HP-related PUD is associated with familial peptic ulcer and the presence of DU. Short-term NSAID use is highly correlated with GU.

Another study by Egbaria et al. from Israel [17], where children have a high prevalence and early acquisition of HP and have easy access to pediatric gastroenterologists and endoscopy, aimed to describe the prevalence and characteristics of PUD in this population. This retrospective study was conducted on 651 diagnostic endoscopies performed over the time period January 2003-May 2006. PUD was regarded as erosive gastritis/duodenitis or ulcer in either the stomach or duodenum. HP status was assessed using rapid urease test and gastric biopsies. PUD was detected in $22.5 \%$ patients (ulcers $6.8 \%$, erosions $15.7 \%$ ). HP was positive in $66.3 \%$ of patients and appeared to be common after the age of 10 years, with gastric PUD presenting much earlier than duodenal disease. Most of the HP PUD were idiopathic and improved symptomatically on PPI treatment. $43 \%$ of patients with PUD were either immigrants from the former Soviet Union or of Israeli Arab origin. In conclusion, PUD appeared to be common in the selected population with a relatively high incidence of gastric PUD. Non-HP PUD 
comprised approximately a third of all PUD, and it was mostly idiopathic and appeared earlier than HP associated PUD.

A Spanish work by Velayos et al. [24] investigated the accuracy of the urea breath test (UBT) performed immediately after emergency endoscopy in peptic ulcer bleeding (PUB). Clinical and epidemiological factors were moreover analyzed in order to study their influence on the accuracy of the early UBT. Early UBT was collected from the 74 patients included. In $71.6 \%$ of the patients, a delayed UBT was obtained. Comparing concordance between the two tests, an accuracy of $83 \%$ for the early UBT was attested. Sensibility and specificity were 86.36 and $66 \%$, respectively, with a positive predictive value of $92.68 \%$ and negative predictive value of $50 \%$ (Kappa index $=0.468$; $P=0.0005$; CI: 95\%). No influence of epidemiological factors, clinical presentation, drugs, times to gastroscopy, Forrest classification, endoscopic therapy, haemoglobin, and urea levels was attested over the accuracy of early UBT. Considering the low negative predictive value for early UBT, a delayed test is mandatory for all negative cases.

A study from Saudi Arabia by El Mouzan and Abdullah [18] aimed to investigate the epidemiology and pattern of the PUD condition in Saudi Arabia. Over a period of 10 years (from 1993 to 2002), 24 (5\%) children out of 521 who presented with upper gastrointestinal tract symptoms were diagnosed by endoscopy to have PUD. The most common presentation was chronic abdominal pain (63\%), followed by vomiting associated with abdominal pain in $4 \%$. Hematemesis and melena occurred in $13 \%$ and $8 \%$, respectively. There were 20 duodenal $(92 \%)$ and four gastric ulcers. Histopathology results of antral biopsies all detected antral gastritis, with detection of HP in $87 \%$ of the specimens. The study concludes that in Saudi children, peptic ulcer is a rare cause of upper GI symptoms, it is mostly observed in boys and often associated with HP antral gastritis.

Among non-HP non-NSAID-related PUD, portal hypertension has been demonstrated to increase the risk for duodenal ulcer in adults. A case-control study from Taipei by Hung et al. [25] investigated the prevalence and characteristics of DU in 80 children with portal hypertension enrolled from January 1997 to December 2001. At upper-GI endoscopic evaluation, the prevalence of DU was significantly higher in children with portal hypertension than in children with digestive symptoms only $(P=0.017)$. DU was more common and appeared earlier in children with a history of variceal bleeding. The presence of DU was independent of the severity of liver disease, HP infection, and serum gastrin level. The authors concluded and suggested to screen patients with gastrointestinal bleeding for DU even in the presence of esophageal varices.

A connection between celiac disease (CD) and non-HP non-NSAID-related PUD was attested by the experience of Levine et al. from Israel [26], who retrospectively reviewed all the endoscopies performed for children and young adults diagnosed with CD between January 2004 and October 2008. Twelve percent (males $52 \%$, females $48 \%$ ) of 240 enrolled patients with diagnosis of $\mathrm{CD}$ had an endoscopic diagnosis of
PUD. 79\% of the PUD patients were HP negative. Duodenal PD was noted in $76 \%$ of $\mathrm{CD}$ patients, and $73 \%$ were $\mathrm{HP}$ negative. Gastric PD was noted in $28 \%$ of CD patients, and $87 \%$ were HP negative. The PUD group was significantly older at diagnosis $(P<0.001)$ compared to the whole CD group. The authors concluded that PUD is not uncommon in the presentation of $\mathrm{CD}$ and is more likely to be found in the second decade of life. CD should be included in the differential diagnosis of patients with non-HP PUD, and routine $\mathrm{CD}$ serology and small bowel biopsy in patients with unexplained PUD should therefore be performed.

An American study by Sarkar et al. [27] analyzed the complications in patients after intestinal transplantation including the occurrence of ulcers in the native or transplanted gastrointestinal tract. Among 112 patients with intestinal transplantation, ulcer formation was endoscopically detected in 11 patients. At the histological examination, the most common changes in the ulcers were compatible with posttransplant lymphoproliferative disorder (PTLD), acute rejection, and viral infections. Some patients were treated for rejection based on the changes within the mucosa outside the ulcer bed, and they responded with resolution of the ulcers. These findings demonstrate that PTLD and acute rejection are the most common causes of chronic ulcer formation in patients with intestinal transplantation and confirm the importance of collecting biopsy samples simultaneously from both the ulcer edge and intervening mucosa.

An Italian case report by Lionetti et al. [28] highlights PUD as a possible threatening GI complication in children with neurofibromatosis type 1 (NF1) and primary ciliary dyskinesia (PCD). The upper gastrointestinal endoscopy performed revealed the presence of multiple gastric ulcers which scarred after the long-term administration of a proton pump inhibitor, but recurred after the suspension. Laboratory and imaging studies excluded Zollinger-Ellison syndrome and other known causes of PUD, suggesting a potential role of NF1 itself and PCD in developing of recurrent PUD. As an early diagnosis of PUD is vital for patient survival, gastric protection before complications arise is to be considered for patients with diagnosis of NF1 and/or PCD.

\subsection{Evidences on Available Treatments for Peptic Ulcer Disease.} When the underlying cause of PUD is addressed, the prognosis is excellent [29]. With regard to NSAID related ulcers, the incidence of perforation is approximately $0.3 \%$ per patient year, and the incidence of obstruction is approximately $0.1 \%$ per patient year. Combining both duodenal ulcers and gastric ulcers, the rate of any complication in all groups combined is approximately $1-2 \%$ per ulcer per year. The mortality rate for PUD is approximately 1 death per 100.000 cases.

HP related ulcers are treated with a course of antibiotics to eradicate the bacterium from the GI tract, as well as with drugs to reduce stomach acid and protect the stomach lining [6].

In 2011 the European and North American Societies of Pediatric Gastroenterology Hepatology and Nutrition (ESPGHAN and NASPGHAN) emitted evidence-based guidelines for managing HP in children [12]. For children 
with HP infection without PUD, treatment should be considered by the clinician evaluating the potential advantages and the risk factors of a developed chronic infection with bad consequences. follows:

The first line recommended therapies are [6-12] as

(i) standard triple therapy with proton pump inhibitorPPI (1-2 mg/kg/day), ampicillin-AMPC (50 mg/kg/ day), clarithromicin-CAM (20 mg/kg/day), or metronidazole-MNZ (20 mg/kg/day);

(ii) bismuth-based quadruple therapy: bismuth subsalicylate $(8 \mathrm{mg} / \mathrm{kg} /$ day $)$, AMPC $(50 \mathrm{mg} / \mathrm{kg} /$ day $)$, and MNZ (20 mg/kg/day);

(iii) sequential therapy: PPI (1-2 mg/kg/day), AMPC ( $50 \mathrm{mg} / \mathrm{kg} / \mathrm{day}$ ) for 5 days, then PPI (1-2 mg/kg/day), CAM (20 mg/kg/day), and MNZ (20 mg/kg/day) for 5 days.

As for adults, an antibiotic susceptibility test should be performed whenever a high resistance rate $(>20 \%)$ in the area is known. Once treatment is completed, a non invasive test should be done to ensure eradication [12].

NSAIDs related ulcers are treated by stopping the causative medications, and by administering drugs that promote healing of the stomach lining.

Stress ulcers, erosions of the stomach and duodenum, and upper GI bleeding are well-known complications of critical illness in children admitted to pediatric intensive care units. Severe illness and a decreased gastric $\mathrm{pH}$ are in fact related to an increased risk of gastric ulceration and haemorrhage.

Prophylactic regimens with PPIs have been shown to dramatically reduce the risk of gastric and duodenal ulcers [30]. Maintenance therapy with antisecretory medications (i.e., H2-blockers, PPIs) for 1 year is indicated in high-risk patients.

A systematic review by Reveiz et al. [31] aimed to identify and evaluate the quality of evidence supporting prophylactic use of treatments for stress ulcers and upper gastrointestinal bleeding. When pooling two randomized controlled trials, treatment was significantly more effective in preventing upper gastrointestinal bleeding (macroscopic or important bleeding) compared with no treatment. No significant difference was seen in death rates among the two groups. The rate of pneumonia was not significantly different when comparing treatment and no treatment in one study. When comparing ranitidine with no treatment, significant differences were found in the proportion of mechanically ventilated children with normal gastric mucosal endoscopic findings by histological specimens.

No significant differences were found when comparing different drugs (omeprazole, ranitidine, sucralfate, famotidine, and amalgate), doses, or regimens for main outcomes (deaths, endoscopic findings of erosion or ulcers, upper gastrointestinal bleeding, or pneumonia).

A multicenter, prospective, cross-sectional observational study by Araujo et al. from Brazil [32] evaluated the use of stress ulcer prophylaxis in patients admitted to five pediatric intensive care units (PICUs) in Porto Alegre. Medical records of 398 patients (57\% males) admitted from April 2006 to February 2007 were reviewed. The mean age was 16 months (IQR 4-65) and respiratory illness was the main reason for admission $(32.7 \%)$. Most patients received stress ulcer prophylaxis $(77.5 \%)$. Ranitidine was the most commonly used drug $(84.5 \%)$. Evidence of minor gastrointestinal bleeding was found in 3\% of patients; none had clinically significant bleeds. The authors concluded that administration of stress ulcer prophylaxis is a common practice in the participating PICUs, with ranitidine the most commonly used drug. Among the various rationales provided, mechanical ventilation and informal routine use were the most prevalent.

Another Chinese study by $\mathrm{Wu}$ [33] investigated and proved the efficacy and safety of famotidine treatment for stress ulcers in 54 neonates with stress ulcers enrolled from 2001 to 2006.

After 24 hours of famotidine treatment, hematemesis and vomiting ceased in $96.3 \%$ of patients. Clinical symptoms disappeared in all patients 48 hrs after famotidine treatment. Gastric $\mathrm{pH}$ value increased after famotidine treatment from $2.07 \pm 0.22$ (before treatment) to 5.01-5.15 $(P<0.01)$. Famotidine treatment did not lead to abnormal respiration, heart rate, and blood pressure. Loss of appetite, nausea, vomiting, diarrhoea, constipation, and rashes were not seen in this study whereas significant differences in white cell count, platelet count, and hepatic enzyme levels before and after famotidine treatment were attested.

A prospective study from the US by Tofil et al. [34] aimed to assess gastric $\mathrm{pH}$ in 48 critically ill pediatric patients (median age 7 years, 5 months; range 1 month to 19 years) with a gastric tube in place, receiving intravenous stress ulcer medication (either ranitidine or a proton pump inhibitor) and no enteral nutrition. All patients were intubated. Ten percent of 28 trough $\mathrm{pH}$ measurements in the twice daily proton pump inhibitor group were more acidic than $\mathrm{pH} 4$ versus $40 \%$ of 60 in the ranitidine group, and $40 \%$ of 56 in the once daily proton pump inhibitor group $(P=0.02)$. Four percent of 27 peak $\mathrm{pH}$ measurements in the twice daily proton pump inhibitor group were more acidic than $\mathrm{pH} 4$ versus $20 \%$ of 61 in the ranitidine group, and $16 \%$ of 56 in the once daily proton pump inhibitor group $(P=0.12)$. Three patients (6\%; 95\% CI, 0.51\%-16\%) developed upper gastrointestinal bleeding, and 4 patients (8\%; 95\% CI, 0\%$13 \%$ ) developed ventilator-acquired pneumonia. This study attests that many critically ill pediatric patients receiving stress ulcer prophylaxis have a trough or peak gastric $\mathrm{pH}$ more acidic than 4 .

Emergency GI endoscopy for upper-GI hemorrhage leads to identification of the bleeding lesion in more than $80 \%$ of cases [35]. The endoscopic assessment should ideally be performed within 6-12 hours after stabilization of the patient. In pediatric patients, gastric and duodenal ulcers are the main indications for endoscopic treatment of nonvariceal GI haemorrhage [36].

Endoscopically visible vessels are usually associated with an organizing clot in the artery close to the ulcer base [35]. Upper GI tract haemorrhage can be endoscopically treated 
using either thermal methods (including laser, heater probe, monopolar or multipolar probes, and plasma argon coagulation) or nonthermal methods (injection of suitable vasoconstrictive or sclerosing agents). Both methods appear to be equally effective but injection is generally preferred because it is cheaper and can be done using a small endoscope [35].

The agents used for injection therapy include dilute epinephrine $(1: 10.000)$, sclerosants, thrombin, and fibrin sealant [35].

Injection therapy with dilute epinephrine reduces the need for surgical intervention and blood transfusion [35]. Submucosal injection of epinephrine causes local vasoconstriction, platelet aggregation, and also volume tamponade. In actively bleeding ulcers, this promptly induces a haemostasis preceded by mucosal oedema and blanching. Since epinephrine does not induce vessel thrombosis, bleeding may recur [35].

Sclerosants such as polidocanol and sodium tetradecyl sulfate cause tissue necrosis and ulceration: they are used in adults whereas they are not generally used in children, given some reports of fatal gastric necrosis [35].

Thrombin and fibrin sealant injections are used in adults but, since the high viscosity of fibrinogen requires a very wide endoscopic channel, the technique is not performed on children [36].

Metallic clips are also available for endoscopic haemostasis of bleeding ulcers, even though the procedure can be technically difficult for ulcers localised in the posterior wall of the duodenal bulb as well as for those localised in the lesser curvature [35].

Thermal devices using contact, include heater probes which are available in two sizes, even though only the smaller type can be used in children aging less than 12 years of age [35].

Thermal devices which do not require contact include argon plasma coagulation which is effective for both oozing and pulsatile arterial bleeding. The technique is safe provoking superficial effects only, with a low risk of perforation; it consists, in children, in the use of a $2.3 \mathrm{~mm}$ diameter flexible delivery catheter filled with argon gas which is passed through the operating channel and the tip of the probe is placed closed to the lesion but without touching it; a coagulative gas flow is then activated [35].

The indications for urgent surgery include failure to achieve haemostasis endoscopically, recurrent bleeding despite endoscopic attempts at achieving haemostasis, and perforation.

A retrospective and prospective hospital-based study in Ghana by Dakubo et al. [36] aimed to determine the epidemiology of and define the morbidity and mortality factors following emergency surgery for 326 patients (range 4-87 years) with perforated PUD. The incidence of PUD in this population was of $5 \%$, and overall accounted for $4.6 \%$ of acute abdomen. Comorbid conditions were present in $18.2 \%$ of cases. Ulcerogenic substance intake was in $67 \%$ patients. $46.2 \%$ patients reported to hospital within 24 hours of perforation. Simple closure with omental patch was performed in $94.3 \%$, truncal vagotomy and drainage in $3.2 \%$, and Billroth II partial gastrectomy in seven $2.2 \%$. Postoperative complications occurred in 19\%; overall mortality was $11 \%$. This study concluded that perforated PUD is emerging as a frequent cause of acute abdomen and affects the youth commonly. A logistic regression analysis of data showed that age of 60 years and above, duration of perforation for more than 24 hours before admission, alcohol intake and resectional surgery were the variables that showed statistical significance in predicting postoperative morbidity and/or mortality.

Another study by Wong et al. from Hong Kong [37] aimed to determine the efficacy, safety, and outcome of 132 children and adolescents undergoing minimally invasive treatment of bleeding and perforation complicating PUD, within the time period from of January 1999 to February 2006. Among 30 children with significant endoscopic stigma of recent haemorrhage, primary endoscopic hemostasis was achieved in all cases except one. Seventeen patients with perforation underwent laparoscopic patch repair. Four patients were converted to open repair because of technical difficulty and the large size of the ulcer. All patients had a course of proton pump inhibitors postoperatively. Ninety percent of patients had HP infestation and were treated with triple therapy; two patients defaulted triple therapy and presented later with recurrent ulcer bleeding. All others remained asymptomatic on followup (average 32.6 months). This study concluded that endoscopic hemostasis of bleeding peptic ulcer is effective and safe in children. With stringent criteria, laparoscopic patch repair of perforation can be applied safely to most pediatric patients. Eradication of HP and subsequent antiulcer medication are integral in the management of complicated PUD.

\section{Conclusions}

PUD in children is reported worldwide with an estimated frequency of $8.1 \%$ in Europe and of $17.4 \%$ in the US, thus remaining relatively rare as compared with adults. PUD mainly occurs in the second decade of life and the most common identified causes in the pediatric age are HP infection and the use of NSAIDs. Other stressful events (i.e., shock, sepsis, burnings, major trauma, intracranial hypertension, surgical procedures, and chronic diseases) can provoke acute gastric ulcers, also in children. Associations of PUD with celiac disease, portal hypertension, intestinal transplantation, and neurofibromatosis are described as well in the pediatric age. An hereditary component is supported by the evidence that more than $20 \%$ of patients have a family history of duodenal ulcers; increased basal acid output and maximal acid output and accelerated gastric emptying are often observed within this subgroup of patients. Alarm symptoms supporting the suspect of GI bleeding or ulcer perforation include sudden, sharp abdominal pain, black or bloody stools, and bloody or coffee-like vomits. Diagnostic examinations is based on rapid urease tests and stool tests for HP detection, and upper-gastrointestinal (GI) tract endoscopy.

When the underlying cause of PUD is addressed, the prognosis is excellent; combining both duodenal ulcers and 
gastric ulcers, the rate of any complication in all groups combined is approximately $1-2 \%$ per ulcer per year in the general population. The mortality rate for PUD is approximately 1 death per 100.000 cases.

HP related ulcers in the children are treated according to the 2011 Evidence Based Guidelines emitted by the European and North American Societies of Pediatric Gastroenterology Hepatology and Nutrition (ESPGHAN and NASPGHAN) emitted evidence-based guidelines for managing HP in children. The first line recommended that therapies comprehend the standard triple therapy, the bismuth-based quadruple therapy, and the sequential therapy.

NSAIDs related ulcers are treated by stopping the causative medications and by administering drugs that promote healing of the stomach lining.

Stress ulcers, erosions of the stomach and duodenum, and upper-GI bleeding are well-known complications of critical illness in children admitted to pediatric intensive care units. Adequate prophylactic regimens have been shown to dramatically reduce the risk of gastric and duodenal ulcers and should therefore be routinely administered within this subgroup of patients.

\section{References}

[1] C. De Giacomo, P. L. Bacchini, M. Lombardi et al., "La patologia gastrica," in L'endoscopia Digestiva in età Pediatrica e Giovanile, G. L. De Angelis, Ed., pp. 49-68, EMSI, Rome, Italy, 2002.

[2] C. Imrie, M. Rowland, B. Bourke, and B. Drumm, "Is Helicobacter pylori infection in childhood a risk factor for gastric cancer?" Pediatrics, vol. 107, no. 2, pp. 373-380, 2001.

[3] M. L. Schubert and D. A. Peura, "Control of gastric acid secretion in health and disease," Gastroenterology, vol. 134, no. 7, pp. 1842-1860, 2008.

[4] N. Gallo, C. F. Zambon, F. Navaglia et al., "Helicobacter pylori infection in children and adults: a single pathogen but a different pathology," Helicobacter, vol. 8, no. 1, pp. 21-28, 2003.

[5] U. Blecker and B. D. Gold, "Gastritis and peptic ulcer disease in childhood," European Journal of Pediatrics, vol. 158, no. 7, pp. 541-546, 1999.

[6] M. Gasparetto, M. Pescarin, and G. Guariso, "Helicobacter pylori eradication therapy: current availabilities," ISRN Gastroenterology, vol. 2012, Article ID 186734, 8 pages, 2012.

[7] A. Capretta, L. Da Dalt, T. Zangardi et al., "Upper gastrointestinal bleeding after ingestion of Ibuprofen. National Congress SIGENP Abstracts," Digestive and Liver Disease, vol. 39, article A75, 2007.

[8] L. Bak-Romaniszyn, S. Wojtuń, J. Gil, and I. Płaneta-Małecka, "Peptic ulcer disease etiology, diagnosis and treatment," Polski Merkuriusz Lekarski, vol. 17, no. 1, pp. 128-132, 2004.

[9] W. D. Chey and B. C. Y. Wong, "American College of Gastroenterology guideline on the management of Helicobacter pylori infection," American Journal of Gastroenterology, vol. 102, no. 8, pp. 1808-1825, 2007.

[10] G. Guariso, D. Basso, C. F. Bortoluzzi et al., "GastroPanel: evaluation of the usefulness in the diagnosis of gastroduodenal mucosal alterations in children," Clinica Chimica Acta, vol. 402, no. 1-2, pp. 54-60, 2009.
[11] D. Basso, C. F. Zambon, D. P. Letley et al., "Clinical Relevance of Helicobacter pylori cagA and vacA gene polymorphisms," Gastroenterology, vol. 135, no. 1, pp. 91-99, 2008.

[12] S. Koletzko, N. L. Jones, K. J. Goodman et al., "Evidence-based guidelines from ESPGHAN and NASPGHAN for Helicobacter pylori infection in children," Journal of Pediatric Gastroenterology and Nutrition, vol. 53, no. 2, pp. 230-243, 2011.

[13] W. Heldwein, J. Schreiner, J. Pedrazzoli, and P. Lehnert, "Is the Forrest classification a useful tool for planning endoscopic therapy of bleeding peptic ulcers?" Endoscopy, vol. 21, no. 6, pp. 258-262, 1989.

[14] H. Shu-Ching, S. Bor-Shyang, L. Shui-Cheng et al., "East etiology and treatment of childhood peptic ulcer disease in taiwan: a single center 9-year experience," African Medical Journal, vol. 86, no. 3, pp. 100-109, 2009.

[15] N. Kalach, P. Bontems, S. Koletzko et al., "Frequency and risk factors of gastric and duodenal ulcers or erosions in children: a prospective 1-month European multicenter study," European Journal of Gastroenterology \& Hepatology, vol. 22, no. 10, pp. 1174-1181, 2010.

[16] K. Brown, P. Lundborg, J. Levinson et al., "Incidence of peptic ulcer bleeding in the US pediatric population," Journal of Pediatric Gastroenterology and Nutrition, vol. 54, no. 6, pp. 733-736, 2012.

[17] R. Egbaria, A. Levine, A. Tamir, and R. Shaoul, "Peptic ulcers and erosions are common in Israeli children undergoing upper endoscopy," Helicobacter, vol. 13, no. 1, pp. 62-68, 2008.

[18] M. I. El Mouzan and A. M. Abdullah, "Peptic ulcer disease in children and adolescents," Journal of Tropical Pediatrics, vol. 50, no. 6, pp. 328-330, 2004.

[19] A. Sawada, "Peptic ulcer in children," Nippon Rinsho, vol. 62, no. 3, pp. 546-550, 2004.

[20] W. Fischbach, P. Malfertheiner, J. C. Hoffmann et al., "S3guideline "Helicobacter pylori and gastroduodenal ulcer disease" of the German Society for Digestive and Metabolic Diseases (DGVS) in cooperation with the German Society for hygiene and microbiology, society for pediatric gastroenterology and nutrition e.V., German Society for rheumatology," Zeitschrift für Gastroenterologie, vol. 47, no. 12, pp. 1230-1263, 2009.

[21] M. Uğraş and E. Pehlivanoğlu, "Helicobacter pylori infection and peptic ulcer in eastern Turkish children: is it more common than known?" Turkish Journal of Pediatrics, vol. 53, no. 6, pp. 632-637, 2011.

[22] J. Sýkora and M. Rowland, "Helicobacter pylori in pediatrics," Helicobacter, vol. 16, supplement 1, pp. 59-64, 2011.

[23] S. H. Berezin, H. E. Bostwick, M. S. Halata, J. Feerick, L. J. Newman, and M. S. Medow, "Gastrointestinal bleeding in children following ingestion of low-dose ibuprofen," Journal of Pediatric Gastroenterology and Nutrition, vol. 44, no. 4, pp. 506-508, 2007.

[24] B. Velayos, L. Fernández-Salazar, F. Pons-Renedo et al., "Accuracy of urea breath test performed immediately after emergency endoscopy in peptic ulcer bleeding," Digestive Diseases and Sciences, vol. 57, no. 7, pp. 1880-1886, 2012.

[25] P. Y. Hung, Y. H. Ni, H. Y. Hsu, and M. H. Chang, "Portal hypertension and duodenal ulcer in children," Journal of Pediatric Gastroenterology and Nutrition, vol. 39, no. 2, pp. 158-160, 2004.

[26] A. Levine, S. Domanov, I. Sukhotnik, T. Zangen, and R. Shaoul, "Celiac-associated peptic disease at upper endoscopy: how common is it?" Scandinavian Journal of Gastroenterology, vol. 44, no. 12, pp. 1424-1428, 2009. 
[27] S. Sarkar, G. Selvaggi, N. Mittal et al., "Gastrointestinal tract ulcers in pediatric intestinal transplantation patients: etiology and management," Pediatric Transplantation, vol. 10, no. 2, pp. 162-167, 2006.

[28] E. Lionetti, R. Francavilla, M. Ruggieri, V. Di Stefano, M. B. Principi, and L. Pavone, "Recurrent peptic ulcer disease in a pediatric patient with type 1 neurofibromatosis and primary ciliary dyskinesia," Minerva Pediatrica, vol. 61, no. 5, pp. 557559, 2009.

[29] M. Bardou, M. Youssef, Y. Toubouti et al., "Newer endoscopic therapies decrease both re- bleeding and mortality in high risk patients with acute peptic ulcer bleeding : a series of metaanalyses," Gastroenterology, vol. 123, article A239, 2003.

[30] K. C. Lai, S. K. Lam, K. M. Chu et al., "Lansoprazole reduces ulcer relapse after eradication of Helicobacter pylori in nonsteroidal anti-inflammatory drug users—a randomized trial," Alimentary Pharmacology and Therapeutics, vol. 18, no. 8, pp. 829-836, 2003.

[31] L. Reveiz, R. Guerrero-Lozano, A. Camacho, L. Yara, and P. A. Mosquera, "Stress ulcer, gastritis, and gastrointestinal bleeding prophylaxis in critically ill pediatric patients: a systematic review," Pediatric Critical Care Medicine, vol. 11, no. 1, pp. 124-132, 2010.

[32] T. E. Araujo, S. M. G. Vieira, and P. R. A. Carvalho, "Stress ulcer prophylaxis in pediatric intensive care units," Jornal de Pediatria, vol. 86, no. 6, pp. 525-530, 2010.

[33] Y. Y. Wu, "Efficacy and safety of famotidine for the treatment of stress ulcers in neonates," Chinese Journal of Contemporary Pediatrics, vol. 10, no. 5, pp. 593-595, 2008.

[34] N. M. Tofil, K. W. Benner, M. P. Fuller, and M. K. Winkler, "Histamine 2 receptor antagonists vs intravenous proton pump inhibitors in a pediatric intensive care unit: a comparison of gastric pH," Journal of Critical Care, vol. 23, no. 3, pp. 416-421, 2008.

[35] J. F. Mougenot, D. Belli, and S. Cadranel, "Endoscopic therapy for nonvariceal bleeding," in Pediatric Gastrointestinal Endoscopy Textbook and Atlas, H. S. Winter, M. S. Murphy, J. F. Mougenot et al., Eds., pp. 137-141, BC Decker, Hamilton, Canada, 2006.

[36] J. C. Dakubo, S. B. Naaeder, and J. N. Clegg-Lamptey, "Gastroduodenal peptic ulcer perforation," Zhongguo Dang dai er ke Za Zhi, vol. 10, no. 5, pp. 593-595, 2008.

[37] B. P. Y. Wong, N. S. Y. Chao, M. W. Y. Leung, K. W. Chung, W. K. Kwok, and K. K. W. Liu, "Complications of peptic ulcer disease in children and adolescents: minimally invasive treatments offer feasible surgical options," Journal of Pediatric Surgery, vol. 41, no. 12, pp. 2073-2075, 2006. 


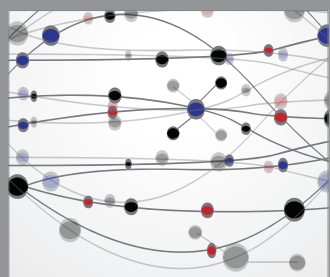

The Scientific World Journal
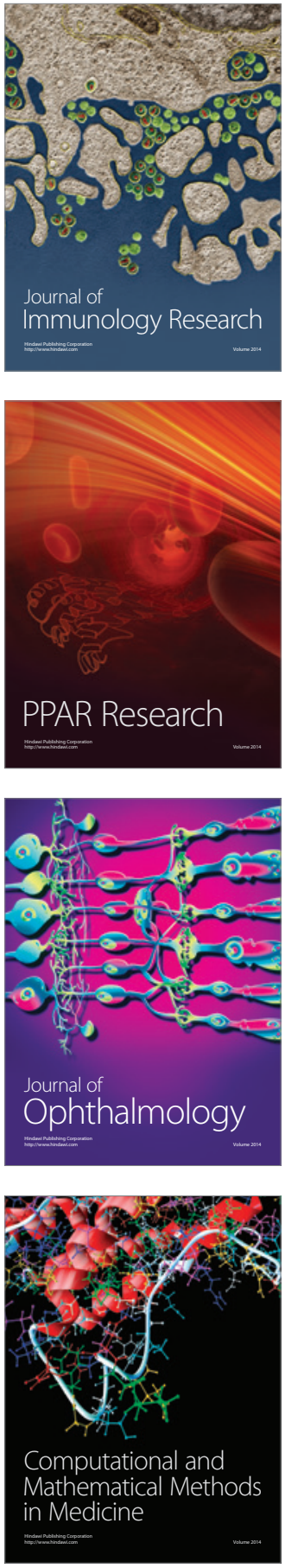

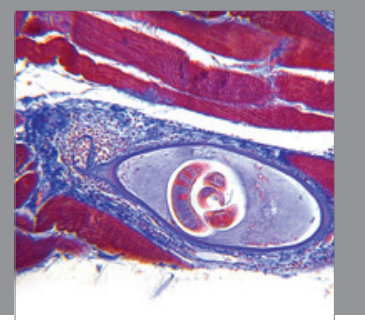

Gastroenterology

Research and Practice
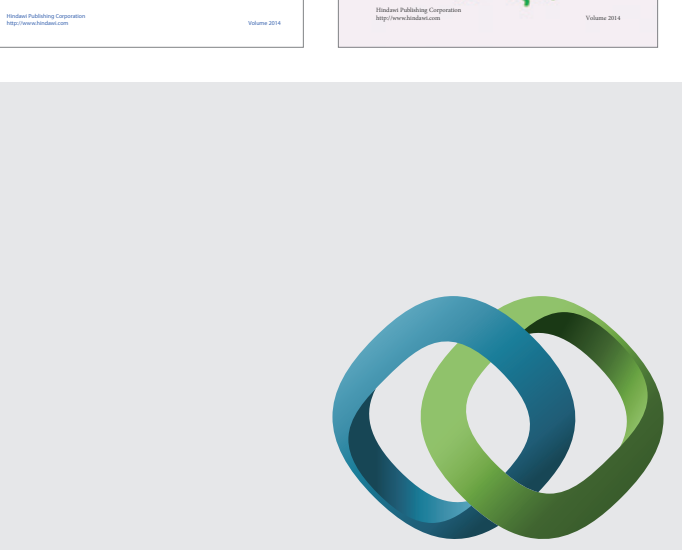

\section{Hindawi}

Submit your manuscripts at

http://www.hindawi.com
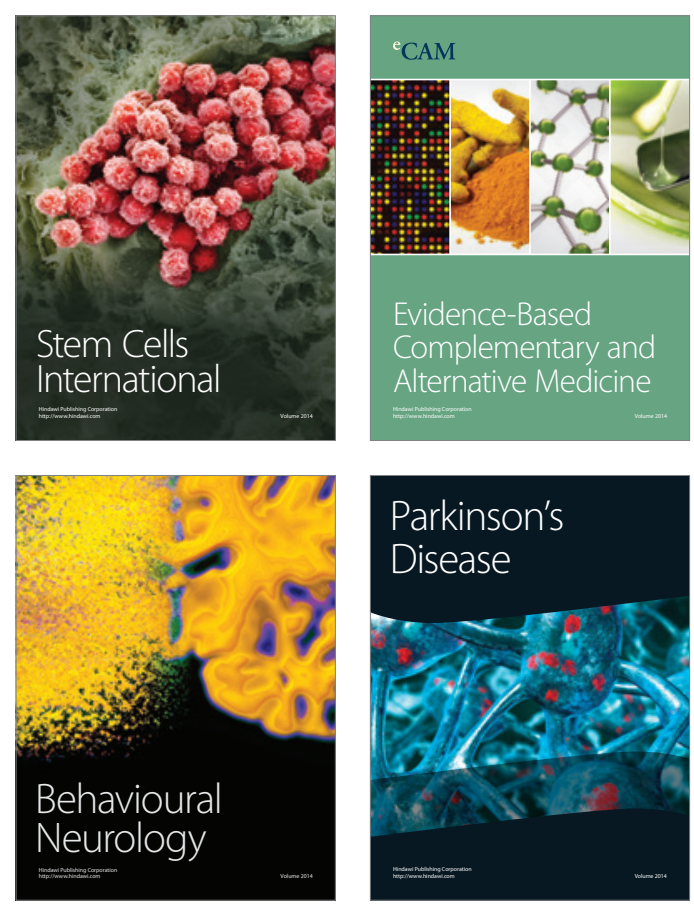

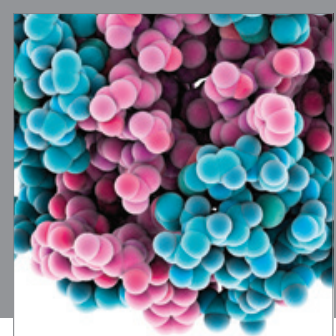

Journal of
Diabetes Research

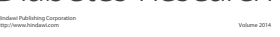

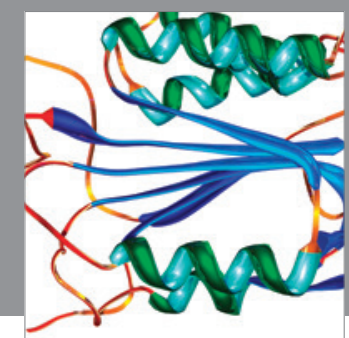

Disease Markers
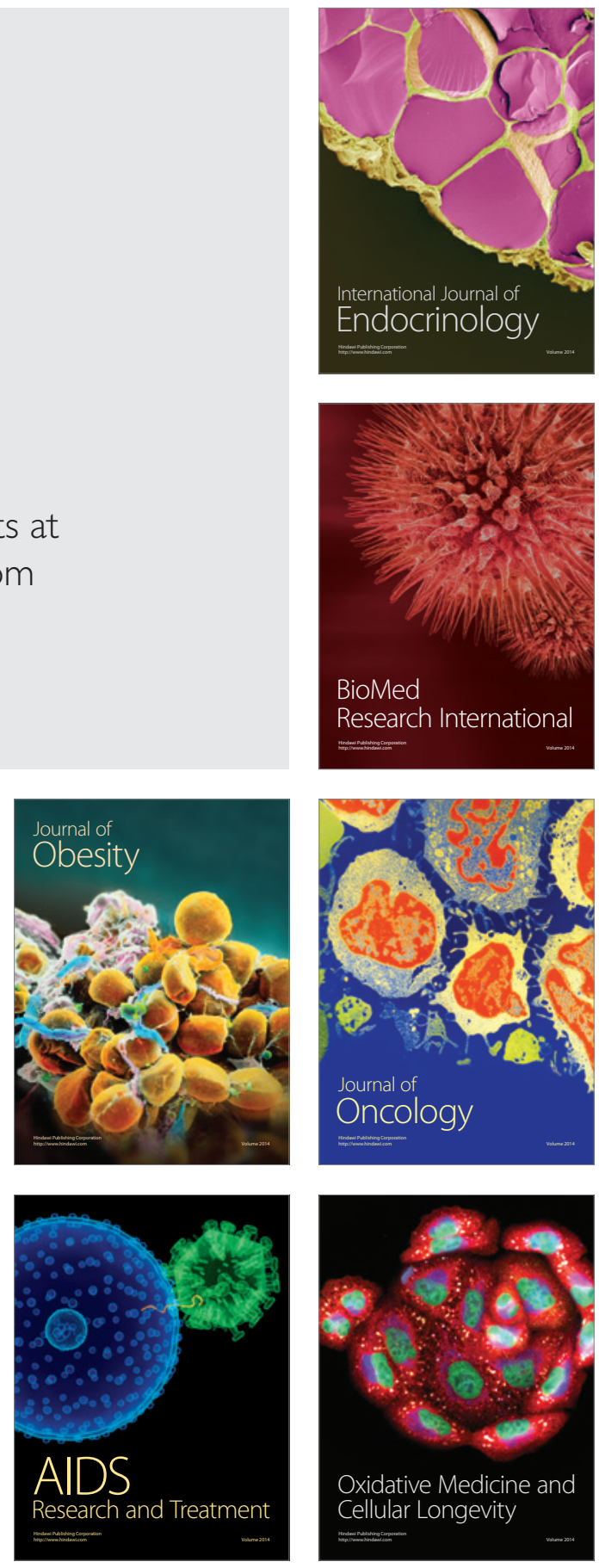